\title{
Supporting Material: Development of effective quantum mechanical/molecular mechanical (QM/MM) methods for complex biological processes
}

${ }^{1}$ Demian Riccardi, ${ }^{1}$ Patricia Schaefer, ${ }^{1}$ Yang Yang, ${ }^{1}$ Haibo Yu

${ }^{1}$ Nilanjan Ghosh, ${ }^{1}$ Xavier Prat-Resina, ${ }^{2}$ Peter König, ${ }^{3}$ Guohui Li ${ }^{4}$ Dingguo Xu, ${ }^{4} \mathrm{Hua}$ Guo, ${ }^{2}$ Marcus Elstner and ${ }^{1}$ Qiang Cui*

${ }^{1}$ Department of Chemistry and Theoretical Chemistry Institute University of Wisconsin, Madison 1101 University Ave, Madison, WI 53706

${ }^{2}$ Theoretische Physik, Universität Paderborn Warburger Str. 100, 33098 Paderborn, Germany

${ }^{3}$ Department of Pathology, Harvard Medical School, 200 Longwood Ave. MA 02115

${ }^{4}$ Department of Chemistry, University of New Mexico, Albuquerque, NM 87131

Submitted as Feature Article in the Journal of Physical Chemistry November 2, 2005

\footnotetext{
${ }^{*}$ To whom the correspondence should be addressed: cui@chem.wisc.edu
} 
In the Supporting Materials, we give more details regarding the SCC-DFTB methods used in different applications in the main text. Due to license issues, the specific parameters associated with the developmental SCC-DFTB methods will not be included here but will be published separately in the near future (Elstner, Cui et al., to be published).

\section{$1 p K_{a}$ calculations}

As described in details in Ref. [1], the standard SCC-DFTB method with parameters established by Elstner et al. [2] was used. This was sufficient because the relative $p K_{a}$ values between protein residues and small molecules in solution were computed.

\section{Water autoionization}

In the study of water autoionization, several improvements were developed: (1). including the third-order expansion as described in Eqs. $(14,15)$ of the main text; (2). including a hydrogen-bonding correction term as described in Eq.(16) of the main text; (3). including both third-order and hydrogen-bonding correction terms. In the following, we give more detailed descriptions on what parameters are needed and how their values were derived.

\subsection{Third-order expansion}

As described in the main text, only on-site third-order expansion has been included. This means that only one parameter, the charge derivative of the Hubbard parameter, $\eta_{\alpha \alpha \alpha}$, is needed for each element type. The value of $\eta_{\alpha \alpha \alpha}$ can be computed based on atomic DFT calculations, which we did for elements $\mathrm{O}, \mathrm{N}, \mathrm{C}$ and $\mathrm{H}$. In addition, the value of $\eta_{\alpha \alpha \alpha}$ has 
been adjusted slightly based on the proton affinity (PA) of a series (32) of small molecules of biological relevance. The results for water and small water clusters are shown in Table 1 as examples. Evidently, the improvement in the computed PAs was dramatic going from the standard second-order expansion to the on-site third-order expansion; optimizations of $\eta_{\alpha \alpha \alpha}$ led to small but systematic further improvements, which reflects the transferrability of the $\eta_{\alpha \alpha \alpha}$ values.

\subsection{Hydrogen-bonding correction}

For the hydrogen-bonding correction, the only parameter is the exponent in the damping function $(\zeta$ in Eq. (16)). The value was determined based on the binding energy of small water clusters (Elstner, unpublished). The performance of the method is illustrated for various water clusters in Table 2.

\subsection{Third-order expansion and hydrogen-bonding correction}

When both the third-order expansion and the hydrogen-bonding correction were included, further adjustment is needed because both methods tend to further polarize the molecule compared to the standard SCC-DFTB approach. To ensure both proton affinities and hydrogen-bonding interactions are well reproduced, we optimized the values of $\eta_{\alpha \alpha \alpha}$ and $\zeta$ (totally five parameters) based on a rather extensive set of data that included 32 proton affinities and 22 hydrogen-bonding interactions associated with small molecules of biological interest. Only results for water and water clusters were included in Tables 1 and 2 as illustrations; the full sets of data and the optimized parameters will be published elsewhere 
(Elstner, Cui and co-workers, to be published). Evidently, optimization improved results, especially the hydrogen-bonding energies, in a significant manner.

\section{Carbonic Anhydrase}

In our studies of Carbonic Anhydrase (CA) during the past several years, as the complexity of calculations evolved from sampling water distributions to computing realistic proton transfer pathways, improvements in the SCC-DFTB method became necessary. For sampling active site water distributions and H64 dynamics (Sect. 3.2.1 in the main text), the original SCC-DFTB method was used. For probing the proton transfer pathways, a much larger QM region that includes many water molecules in the active site is necessary. Careful analysis of the MD trajectories and minimum energy path (MEP) calculations revealed unexpected reorientations of QM water molecules as a result of underestimated hydrogen-bonding interactions by the standard SCC-DFTB method. Therefore, the hydrogen-bonding correction in SCC-DFTB (Sect. 2.2. here) was included in the proton transfer studies. We note that the third-order correction was found not necessary in this context because the relative proton affinity of zinc-bound water and His 64 was already well described without the third-order terms; this was confirmed by single point B3LYP/6-311+G(d,p)/MM calculations at structures along proton transfer pathways collected at the SCC-DFTB/MM level. Analysis of the MEP results also revealed that although including the hydrogen-bonding correction had a very minor impact on the reaction energy of the proton transfers it modified the barrier height significantly. It is worth noting that the latter effect was observed only for transition states with multiple protons being transferred; for strict step-wise transfers, the effect of the 
hydrogen-bonding correction on the barrier was also very small. The solution we adopted for this problem was to modify the $\mathrm{O}-\mathrm{H}$ repulsive potential $\left(U_{\alpha \beta}(R)\right.$ in Eq. (14) in the main text). Instead of refitting the entire O-H repulsive potential, a Gaussian function was added as a perturbation; a set of windowing functions were also included to ensure that the Gaussian has the largest effect for $\mathrm{O}-\mathrm{H}$ distances typically found in the barrier region; this was found critical to avoid perturbing hydrogen-bonding energies. Specifically, the O-H repulsive potential was modified by the following expression,

$$
U_{O H}^{R e p}(R)=U_{O H}^{R e p, s t d}(R)+a_{1} \exp ^{-\frac{\left(R-a_{2}\right)^{2}}{a_{3}}} S(R)
$$

with $S(r)$

$$
S(R)= \begin{cases}0, & R \leq r_{o f f 1} \\ \frac{\left(r_{o f f 1}^{2}-R^{2}\right)^{2}\left(r_{o f f 1}^{2}+2 R^{2}-3 r_{o n}^{2}\right)}{\left(r_{o f f 1}^{2}-r_{o n}^{2}\right)^{3}}, & r_{o f f 1}<R \leq r_{o n} \\ \frac{\left(r_{o f f 2}^{2}-R^{2}\right)^{2}\left(r_{o f f 2}^{2}+2 R^{2}-3 r_{o n}^{2}\right)}{\left(r_{o f f}^{2}-r_{o n}^{2}\right)^{3}}, & r_{o n}<R \leq r_{o f f 2} \\ 0, & r_{o f f 2}<R\end{cases}
$$

and

$$
\begin{aligned}
& r_{o f f 1}=r_{o n}-\delta r \\
& r_{o f f 2}=r_{o n}+\delta r
\end{aligned}
$$

The parameters $a_{1}, a_{2}, a_{3}$ and $r_{\text {on }}$ were optimized using a genetic algorithm, [3] while $\delta r$ was chosen to be 0.50 bohr. 
The properties used to parameterize $a_{1}, a_{2}, a_{3}$ and $r_{o n}$ included: (neutral and protonated) water dimer binding energy, water monomer geometry, water monomer/dimer $\mathrm{O}-\mathrm{H}$ vibrational frequencies, and the energetics of the proton transfer in $\mathrm{H}_{5} \mathrm{O}_{2}{ }^{+}$with $\mathrm{R}(\mathrm{OO})$ set to $3.0 \AA$; in addition, a simple gas-phase active site CA model was used which included a "zinc" bound water, 4 bridging water molecules and a 4-methyl imidazole; the "zinc" was treated with a simple point charge of " $+1.25 \mathrm{e}$ " to speed up the optimization process and all other atoms were treated with SCC-DFTB.

In Figure 1, the optimized O-H repulsive potential is compared with the standard set. In Table 3 and Fig.2, various of properties obtained using the fitted repulsive potential are shown, in comparison with the target data. The values for the optimized parameters will be published elsewhere. 


\section{References and Notes}

(1) Riccardi, D.; Schaefer, P.; Cui, Q. J. Phys. Chem. B 2005, 109, 17715-17733.

(2) Elstner, M.; Porezag, D.; Jungnickel, G.; Elsner, J.; Haugk, M.; Frauenheim, T.; Suhai, S.; Seifert, G. Phys. Rev. B 1998, 58(11), 7260-7268.

(3) FORTRAN Genetic Algorithm (GA) Driver. Carroll, D. L. Jan. , 1997.

(4) Linstrom, P. J.; Mallard, W. G. NIST Chemistry WebBook, NIST Standard Reference Database Number 69, March 2003; http://webbook.nist.gov/chemistry/, 2003. 
Table 1. Comparison of proton affinity predicted by different parameterizations of SCCDFTB and high level $a b$ initio calculations for water and water clusters ${ }^{\mathrm{a}}$

\begin{tabular}{|c|c|c|c|c|c|}
\hline \multirow[b]{2}{*}{ Molecules $^{b}$} & \multirow[b]{2}{*}{ G3B3 $^{c}$} & \multicolumn{4}{|c|}{ SCC-DFTB } \\
\hline & & Standard ${ }^{d}$ & $3^{\text {rd }}$-order ${ }^{e}$ & $\begin{array}{c}3^{\text {rd }} \text {-order } \\
\text { Optimized }\end{array}$ & $\begin{array}{c}3^{\text {rd }} \text {-order + } \\
\text { HBond }^{g}\end{array}$ \\
\hline $\mathrm{H}_{2} \mathrm{O}$ & 398.4 & 26.5 & -5.4 & 1.3 & 1.2 \\
\hline $2 \mathrm{H}_{2} \mathrm{O}\left(\mathrm{H}^{+}\right)$ & 200.2 & 6.8 & -1.6 & -0.1 & 0.9 \\
\hline $3 \mathrm{H}_{2} \mathrm{O}\left(\mathrm{H}^{+}\right)$ & 213.4 & 4.2 & -4.6 & -3.1 & -2.8 \\
\hline $4 \mathrm{H}_{2} \mathrm{O}\left(\mathrm{H}^{+}\right)$ & 221.1 & 4.3 & -4.3 & -2.7 & -2.9 \\
\hline $5 \mathrm{H}_{2} \mathrm{O}\left(\mathrm{H}^{+}\right)$ & 226.7 & 3.9 & -4.5 & -3.0 & -2.4 \\
\hline $2 \mathrm{H}_{2} \mathrm{O}$ & 375.9 & 19.8 & -3.6 & 1.4 & -2.4 \\
\hline $3 \mathrm{H}_{2} \mathrm{O}$ & 365.0 & 18.5 & -4.6 & 0.4 & -4.8 \\
\hline $4 \mathrm{H}_{2} \mathrm{O}$ & 359.1 & 17.4 & -4.2 & 0.5 & -4.9 \\
\hline \multirow{2}{*}{$5 \mathrm{H}_{2} \mathrm{O}$} & 348.4 & 19.7 & -4.9 & 0.8 & -7.5 \\
\hline & \multicolumn{5}{|c|}{ Error Analysis $^{h}$} \\
\hline MAXE & & 32.8 & -10.8 & 8.3 & -8.7 \\
\hline RMSE & & 11.9 & 4.9 & 3.9 & 4.6 \\
\hline MUE & & 9.4 & 4.3 & 3.2 & 3.9 \\
\hline MSE & & 8.4 & -1.7 & 0.4 & -0.2 \\
\hline
\end{tabular}

a) The proton affinity (PA) of $\mathrm{A}^{-}$is defined as the negative of the enthalpy change of the gas phase reaction $\mathrm{A}_{(\mathrm{g})}^{-}+\mathrm{H}^{+}{ }_{(\mathrm{g})} \rightarrow \mathrm{AH}_{(\mathrm{g})}$. In all calculations carried out here (including the G3B3 calculations), $0 \mathrm{~K}$ electronic energies instead of the room temperature enthalpy were used to calculate PA, without the zero point energy correction. All of the quantities have the unit of $\mathrm{kcal} / \mathrm{mol}$. For G3B3, the absolute PA's are shown; for all other columns, the differences from the G3B3 values are given. Structures were fully optimized at respective levels.

b) All the molecules correspond to the $\mathrm{AH}_{(\mathrm{g})}$ species in the above reaction. Although 32 small molecules were analyzed and used in parameterization, only water and water clusters are included for illustration. The full set of data will be published elsewhere.

c) For calculations involved in G3B3, refer to A. G. Baboul, L. A. Curtiss, P. C. Redfern, and K. Raghavachari, J. Chem. Phys., 1999, 110, 7650-7657 and L. I A. Curtiss, K. Raghavachari, P. C. Redfern, V. Rassolov, and J. A. Pople, J. Chem. Phys., 1998, 109, 7764-7776. Note that thermal contributions were not included here.

d) The value of $141.8 \mathrm{kcal} / \mathrm{mol}$ was used to account for self-interaction energy of the $\mathrm{H}$ atom for all SCC-DFTB calculations.

e) In the $3^{\text {rd }}$-order expansion, the on-site charge derivative of the Hubbard parameter $\left(\eta_{\alpha \alpha \alpha}\right)$ was evaluated for each element type based on atomic DFT calculations (Elstner, not published) and was not optimized for optimal PA values .

f) In the $3^{\text {rd }}$-order expansion, the on-site charge derivative of the Hubbard parameter $\left(\eta_{\alpha \alpha \alpha}\right)$ was optimized for the best PA values for 32 small molecules of biological relevance. A Genetic Algorithm (GA) was applied for the optimization, in which the PA and the root-mean-square-gradient of the G3B3 geometries were included as target data; they were assigned of the weight of 10 and 1, respectively.

g) Both $\eta_{\alpha \alpha \alpha}$ and $\zeta$ in the hydrogen-bonding correction were optimized for the optimal PA and 22 hydrogen-bonding energies (for molecules listed in Table 2). 
h) The error analysis were done for all 32 small molecules used in the parameterization. MAXE refers to error with largest magnitude, defined as sign(err) $\max (|\operatorname{err}|)$; RMSE refers to root-mean-square error, defined as $<(\mathrm{err})^{2}>^{1 / 2}$; MUE refers to mean unsigned error, defined as $<\mid$ err $\mid>$; MSE refers to mean signed error <err $>$, where "err" is the PA difference explained above. 
Table 2. Comparison of binding energies predicted by different parameterizations of SCCDFTB and high level $a b$ initio calculations for various water clusters ${ }^{\mathrm{a}}$

\begin{tabular}{|c|c|c|c|c|c|c|}
\hline & & \multicolumn{5}{|c|}{ SCC-DFTB $^{b}$} \\
\hline Molecules $^{d}$ & $\begin{array}{c}a b \\
\text { initio }^{c}\end{array}$ & Standard & HBond & $3^{\text {rd }}$-order & $\begin{array}{c}3^{\text {rd }} \text {-order } \\
\text { Optimized }\end{array}$ & $\begin{array}{c}3^{\text {rd }} \text {-order }+ \\
\text { HBond }\end{array}$ \\
\hline $2 \mathrm{H}_{2} \mathrm{O}$ & -4.9 & 1.6 & 0.3 & 1.1 & 1.2 & 0.2 \\
\hline $3 \mathrm{H}_{2} \mathrm{O}$ & -15.1 & 5.5 & 0.9 & 3.7 & 4.2 & 0.3 \\
\hline $4 \mathrm{H}_{2} \mathrm{O}$ & -27.4 & 10.3 & 3.5 & 7.6 & 8.2 & 2.7 \\
\hline $5 \mathrm{H}_{2} \mathrm{O}$ & -36.3 & 14.0 & 5.2 & 10.6 & 11.4 & 4.2 \\
\hline $2 \mathrm{H}_{2} \mathrm{O}\left(\mathrm{H}^{+}\right)$ & -33.9 & 4.6 & -0.5 & 6.1 & 5.7 & 2.5 \\
\hline $3 \mathrm{H}_{2} \mathrm{O}\left(\mathrm{H}^{+}\right)$ & -57.3 & 11.1 & 2.6 & 11.8 & 11.7 & 6.3 \\
\hline $4 \mathrm{H}_{2} \mathrm{O}\left(\mathrm{H}^{+}\right)$ & -77.2 & 15.6 & 5.3 & 15.2 & 15.3 & 8.7 \\
\hline $5 \mathrm{H}_{2} \mathrm{O}\left(\mathrm{H}^{+}\right)$ & -91.9 & 20.0 & 6.8 & 18.6 & 19.0 & 10.0 \\
\hline $2 \mathrm{H}_{2} \mathrm{O}\left(-\mathrm{H}^{+}\right)$ & -27.4 & -5.1 & -11.2 & 2.8 & 1.3 & -3.4 \\
\hline $3 \mathrm{H}_{2} \mathrm{O}\left(-\mathrm{H}^{+}\right)$ & -48.6 & -2.4 & -13.8 & 4.6 & 3.3 & -5.7 \\
\hline $4 \mathrm{H}_{2} \mathrm{O}\left(-\mathrm{H}^{+}\right)$ & -66.7 & 1.1 & -13.4 & 8.8 & 7.4 & -3.5 \\
\hline $5 \mathrm{H}_{2} \mathrm{O}\left(-\mathrm{H}^{+}\right)$ & -86.3 & 7.2 & -12.2 & 11.1 & 11.0 & -4.5 \\
\hline $6 \mathrm{H}_{2} \mathrm{O}$ _book & -45.6 & 17.3 & 5.8 & 12.9 & 14.0 & 4.4 \\
\hline $6 \mathrm{H}_{2} \mathrm{O}_{\text {_cage }}$ & -45.8 & 17.1 & 4.3 & 12.3 & 13.4 & 2.3 \\
\hline $6 \mathrm{H}_{2} \mathrm{O}$ _prism & -45.9 & 16.9 & 3.3 & 11.9 & 13.1 & 1.6 \\
\hline \multirow[t]{2}{*}{$6 \mathrm{H}_{2} \mathrm{O}$ _ring } & -44.9 & 17.6 & 6.7 & 13.3 & 14.3 & 5.6 \\
\hline & & \multicolumn{5}{|c|}{ Error Analysis ${ }^{e}$} \\
\hline MAXE & & 20.0/-5.1 & $-13.8 /-11.2$ & 18.6/6.1 & $19.0 / 5.7$ & $10.0 /-6.0$ \\
\hline RMSE & & $10.5 / 3.0$ & $6.4 / 3.0$ & $9.2 / 3.0$ & $9.5 / 2.8$ & $4.4 / 2.0$ \\
\hline MUE & & $8.2 / 2.7$ & $4.9 / 1.9$ & $7.7 / 2.7$ & $7.8 / 2.5$ & $3.6 / 1.5$ \\
\hline MSE & & $7.6 / 2.1$ & $-0.1 /-0.4$ & $7.2 / 2.1$ & $7.4 / 2.2$ & $1.5 / 0.3$ \\
\hline
\end{tabular}

a) In the current work, all binding energies were computed in the gas phase and $0 \mathrm{~K}$ without the zero point energy correction; for water clusters with more than two water molecules, the binding energies are total binding energies relative to isolated monomers. All of the quantities have the unit of $\mathrm{kcal} / \mathrm{mol}$. For the "ab initio" results (see below), the absolute binding energies are given; for SCC-DFTB, differences from the $a b$ initio values are given.

b) See the footnotes of Table 1 for different parameterizations of the SCC-DFTB approach. The additional column labeled as "HBond" indicates adding only the hydrogen-bond correction to the standard SCC-DFTB approach, without any third-order terms or extensive optimizations.

c) For the first 12 molecular systems, the "ab initio" corresponds to the G3B3 method, without the thermal contributions. For the four neutral water hexamers, results from CBS calculations were used (S. Xantheas, C. J. Burnham and R. J. Harrison, J. Chem. Phys., 2002, 116, 1493-1499).

d) Although 22 binding energies were used for the parameterizations, only water clusters are shown for illustrations. " $n \mathrm{H}_{2} \mathrm{O}$ " refer to neutral water cluster; " $n \mathrm{H}_{2} \mathrm{O}\left(\mathrm{H}^{+}\right)$" refer to the protonated water clusters; " $n \mathrm{H}_{2} \mathrm{O}\left(-\mathrm{H}^{+}\right)$" refer to the deprotonated water clusters.

e) The error analyses were done for all $\mathbf{2 2}$ binding energies used in the parameterization. Same notations were used as in Table 1. The numbers before and after the slashes are 
errors in the total binding energy and binding energy per hydrogen-bond (i.e., total binding energy divided by the number of hydrogen bonds in each molecule), respectively. 


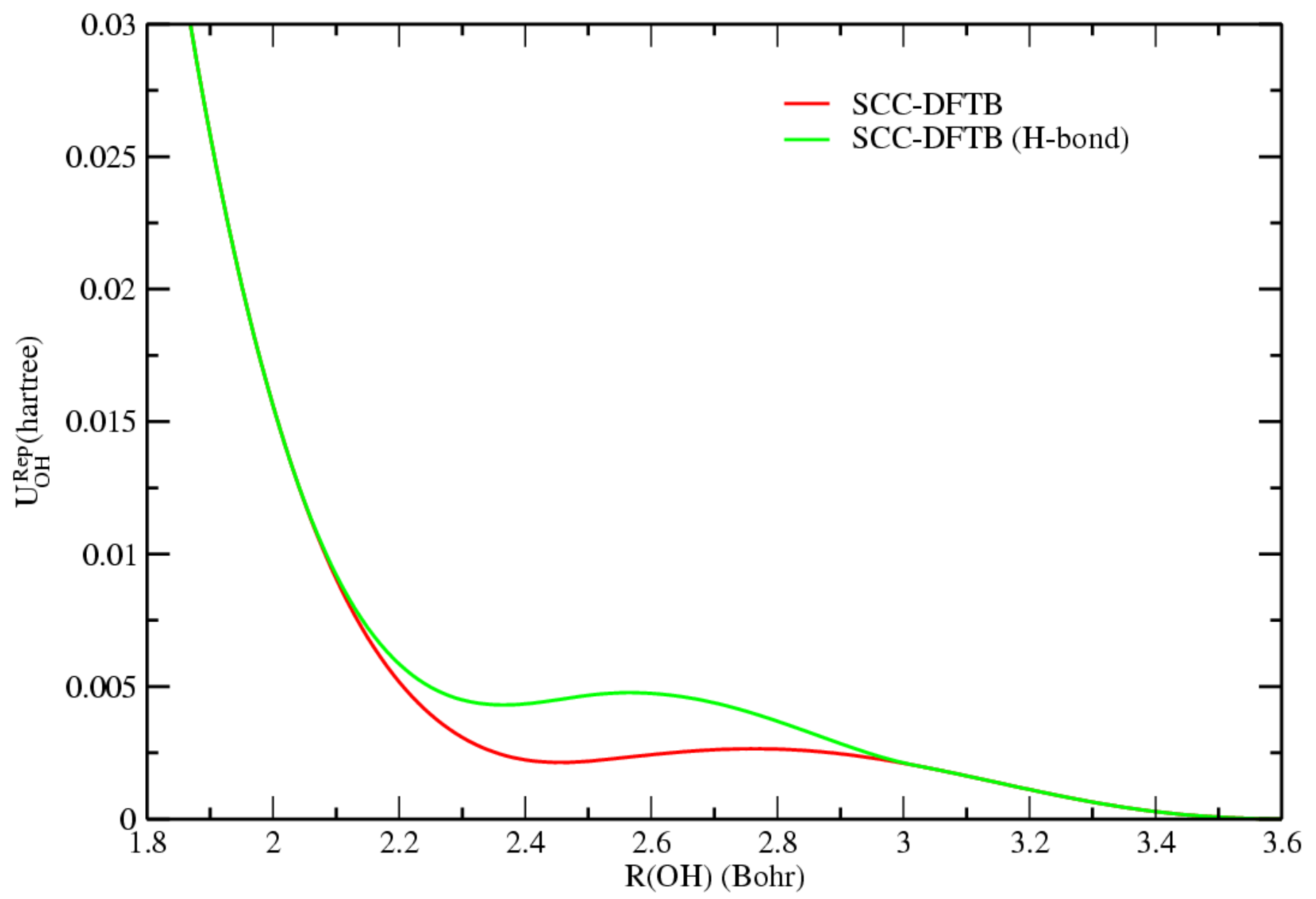

Figure 1. Comparison for the original and modified repulsive potential for the $\mathrm{O}-\mathrm{H}$ atomic pair. 


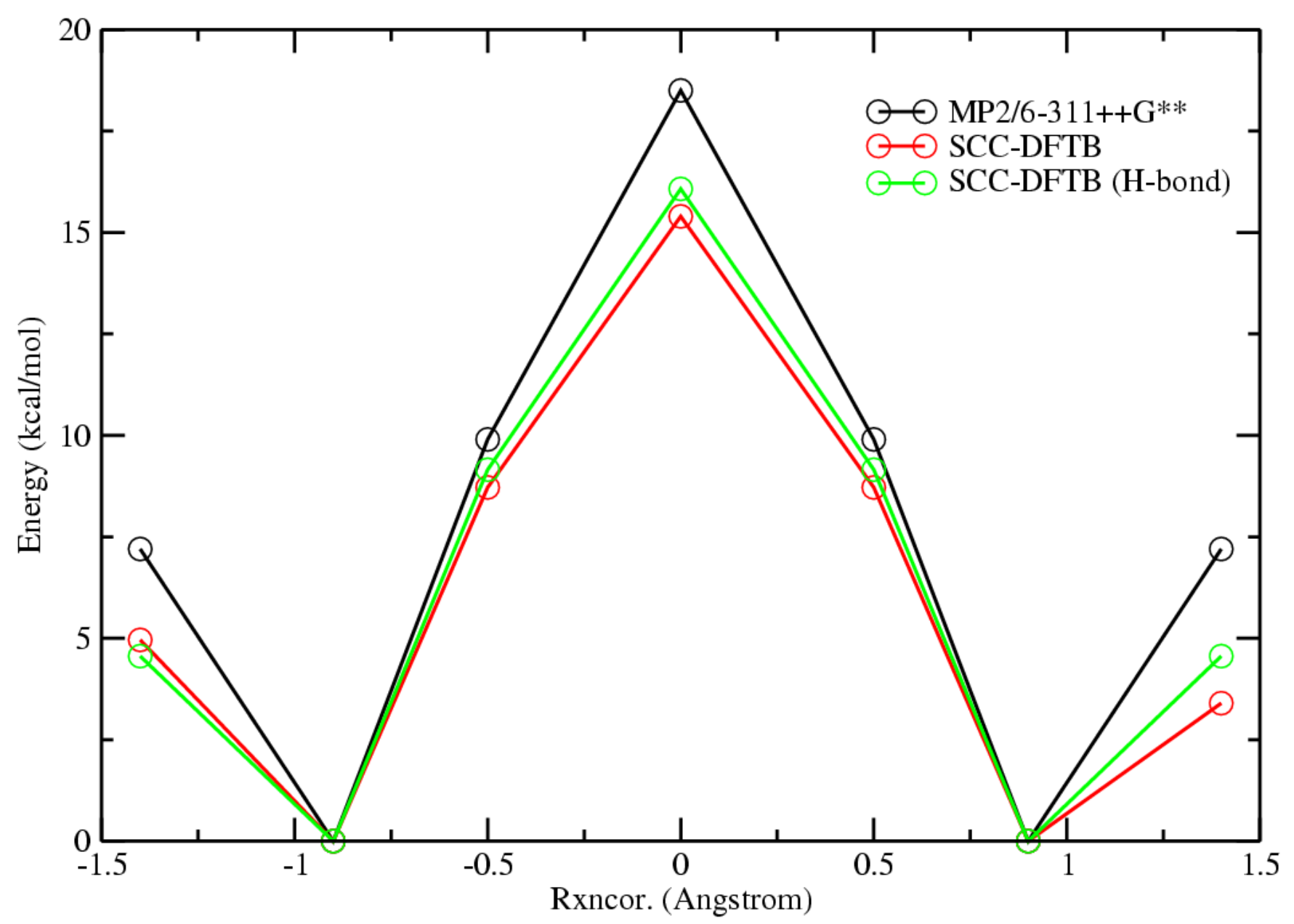

Figure 2. Proton transfer in $(\mathrm{H} 5 \mathrm{O} 2)^{+}$. The distance between the Oxygen atoms is fixed at $3.0 \AA$. The Rxncor coordinate is defined as the anti-sysmetric stretch between the proton and the two Oxygen atoms. 
TABLE 3: Properties used to fit the O-H repulsive potential for the SCC-DFTB method with the hydrogen-bonding correction. Energy in $\mathrm{kcal} / \mathrm{mol}$, distance in $\AA$ and angle in degree, frequency in $\mathrm{cm}^{-1}$.

\begin{tabular}{|c|c|c|c|c|c|c|c|c|c|c|}
\hline & \multicolumn{5}{|c|}{ Proton transfer barrier ${ }^{a}$} & \multirow[t]{2}{*}{$\left(\mathrm{H}_{2} \mathrm{O}\right)_{2}{ }^{b}$} & \multirow[t]{2}{*}{$\left(\mathrm{H}_{5} \mathrm{O}_{2}\right)^{+c}$} & \multicolumn{3}{|c|}{ water geometry $^{d}$} \\
\hline & 1 & 2 & 3 & 4 & 5 & & & $\mathrm{R}(\mathrm{OH})$ & $\mathrm{R}(\mathrm{OH})$ & $\angle \mathrm{HOH}$ \\
\hline Target & 0.0 & 9.1 & 10.7 & 8.6 & 0.1 & -4.9 & -34.4 & 0.957 & 0.957 & 104.5 \\
\hline SCC-DFTB (H-bond) & 0.0 & 9.2 & 10.1 & 10.3 & 1.3 & -4.6 & -33.1 & 0.965 & 0.965 & 108.7 \\
\hline & $\begin{array}{l}\text { ha } \\
\text { mon }\end{array}$ & $\begin{array}{l}\text { monic } \\
\text { mer }\end{array}$ & $\overline{\mathrm{O}-\mathrm{H} \text { vi }}$ & ration & $\begin{array}{l}\text { Ifrequ } \\
\text { mer }\end{array}$ & ency & & & & \\
\hline Target & 3834 & 3943 & 3715 & 3801 & 3879 & 3897 & & & & \\
\hline SCC-DFTB (H-bond) & 3759 & 4027 & 3664 & 3756 & 3965 & 4023 & & & & \\
\hline
\end{tabular}

a: The model included a "zinc" bound water, 4 bridging water molecules and a 4-methyl imidazole; the "zinc" was treated with a simple point charge with " $+1.25 \mathrm{e}$ " and all other atoms were treated with SCC-DFTB. The target values were taken from the standard SCC-DFTB calculations.

b: The target values were taken from G3B3 calculations without zero-point corrections as described in Table 1-2.

c: The target values were taken from G3B3 calculations without zero-point corrections as described in Table $1-2$.

d: The target values were taken from the experimental data [4].

e: The target values for the harmonic frequencies were taken from the experimental data [4]. 